\title{
Conformal Miniaturization of Domains with Low Domain-Wall Energy: Monoclinic Ferroelectric States near the Morphotropic Phase Boundaries
}

\author{
Y. M. Jin, Y. U. Wang, and A. G. Khachaturyan \\ Department of Ceramic and Materials Engineering, Rutgers University, Piscataway, New Jersey 08854, USA
}

\author{
J. F. Li and D. Viehland
}

Department of Materials Science and Engineering, Virginia Tech, Blacksburg, Virginia 24061, USA

(Received 24 April 2003; published 6 November 2003)

\begin{abstract}
A theory is developed for intermediate monoclinic $\left(\mathrm{FE}_{m}\right)$ phases near morphotropic phase boundaries in ferroelectrics of complex oxides. It is based on the conformal miniaturization of stress-accommodating tetragonal domains under the condition of low domain-wall energy density. The microdomainaveraged lattice parameters are determined and attributed to the parameters of an adaptive monoclinic phase. The theory is applied to the temperature, electric field, and compositional dependent $\mathrm{FE}_{m}$ lattice parameters. The predictions of the theory are rigidly obeyed over the entire $\mathrm{FE}_{m}$ stability range.
\end{abstract}

DOI: 10.1103/PhysRevLett.91.197601

PACS numbers: 77.80.-e, 77.84.-s

Neutron and $\mathrm{x}$-ray diffraction investigations of oriented piezoelectric single crystals of $\mathrm{Pb}\left(\mathrm{Mg}_{1 / 3} \mathrm{Nb}_{2 / 3}\right)$ $\mathrm{O}_{3}-x \mathrm{PbTiO}_{3} \quad(\mathrm{PMN}-x \% \mathrm{PT})$ and $\mathrm{Pb}\left(\mathrm{Zn}_{1 / 3} \mathrm{Nb}_{2 / 3}\right)-$ $\mathrm{O}_{3}-x \mathrm{PbTiO}_{3}(\mathrm{PZN}-x \% \mathrm{PT})$ have shown the presence of intermediate monoclinic $\left(\mathrm{FE}_{m}\right)$ states, sandwiched between rhombohedral $\left(\mathrm{FE}_{r}\right)$ and tetragonal $\left(\mathrm{FE}_{t}\right)$ ferroelectric phases near a morphotropic phase boundary (MPB) [1-4]. An explanation of these phases in terms of the conventional Ginzburg-Landau-Devonshire (GLD) theory of homogeneous ferroelectric phases is quite difficult. The homogeneous $\mathrm{FE}_{m}$ phase can be described only if eight terms in the free energy expansion are included and thus eight fitting parameters are introduced [5]. However, the GLD theory cannot explain the observed special relations between the crystal lattice parameters of the $\mathrm{FE}_{m}$ phase discussed in this Letter. Basic-principles calculations (based on an assumption of microscopic homogeneity of the ferroelectric order) have shown that the intermediate phases could be attributed to rotational polarization instabilities [6-8]. These instabilities, in fact, demonstrate that the crystallographic anisotropy of the polarization direction vanishes (or at least is drastically reduced) near the MPB. Accordingly, the domainwall energy will also be dramatically decreased.

The purpose of this Letter is to demonstrate that a homogeneous ferroelectric state is unstable under these special conditions. For very low values of domain-wall energies, the system transforms into a mixed (or adaptive) state $[9,10]$. This adaptive state is inhomogeneous on the nanoscale and homogeneous on the macroscale. The nanoscale microstructure of this adaptive state is a miniaturized microdomain structure determined by the accommodation of the misfit-generated stress and electric field. We show that changes in the microdomain topology result in gradual average symmetry adaptations. Previous experimental studies have shown microdomains on the scale of $10 \mathrm{~nm}$, which gradually change with temperature and composition [11], consistent with the theory.

The conventional ferroelastic microstructure consists of polydomain plates $[12,13]$. Each plate is formed by alternating layers of twin-related domains, as shown in Fig. 1 [14]. The relative thicknesses of the domain layers are adjusted to establish the macroscopic invariance of the habit plane $[12,13]$ and in so doing eliminate longrange stress fields generated by crystal lattice misfits

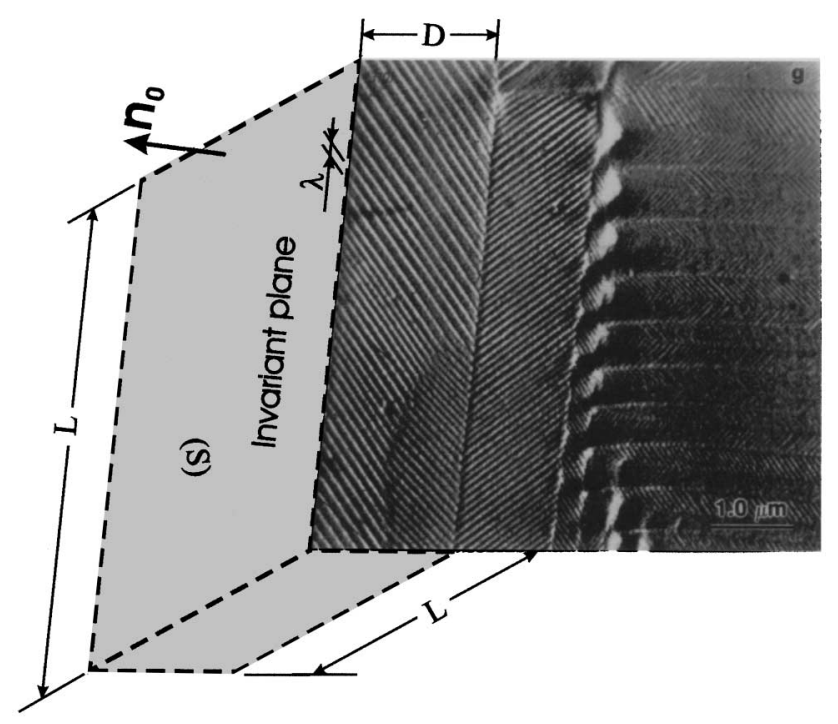

FIG. 1. Dark-field TEM image of a stress-accommodating polydomain structure in a $\mathrm{CuAu}$ alloy [14]. The structure of the adaptive phase has the same morphology but is conformally miniaturized to reach nano- or subnanoscale. Then white and black stripes become microdomains that are "invisible" to the usual diffraction measurement and the macroplates become macrodomains that are perceived as domains of the "homogeneous" monoclinic phase (adaptive phase). 
[15-17]. This requires the domain-averaged stressfree transformation strain of each plate to be an invariant plane strain (IPS), where the invariant plane is parallel to the habit plane. Stress-accommodating domain structures typical of ferroelastics will occur for any displacive transformation, which can form a domain-averaged transformation strain that is an IPS. The presence of polarization in structural domains will result in a formation of the head-to-tail pattern of the $90^{\circ}$ ferroelectric domains. It does not generate spatial charges and thus automatically provides electrostatic energy minimization.

Minimization of the sum of the strain and interfacial energies produces structural domains assembled in polydomain plates shown in Fig. 1. The typical domain size $\lambda_{0}$ is related to the thickness of the polydomain plate $D$ and the domain-wall energy density $\gamma$ :

$$
\lambda_{0}=\beta \sqrt{\frac{\gamma}{\mu \varepsilon_{0}^{2}} D},
$$

where $\beta$ is a dimensionless constant, $\mu$ is the shear modulus, and $\varepsilon_{0}$ is the twinning strain $[15,16]$. Analogously, the typical thicknesses of ferroelectric and ferromagnetic domains in a plate of thickness $D$ are also proportional to $\sqrt{\gamma D}$.

The basic assumption of our theory is abnormally small domain-wall energy density $\gamma$. It follows from Eq. (1) that if $\gamma \rightarrow 0$, then $\lambda_{0} \rightarrow 0$ as well, i.e., a reduction of $\gamma$ results in domain miniaturization. If $\gamma$ is sufficiently small, the domain pattern shown in Fig. 1 will be conformally miniaturized to the nanoscale. Then the plates consisting of nanoscale microdomains can be regarded as macrodomains of the adaptive state. These tetragonal microdomains cannot be observed by x-ray diffraction if the microdomain thickness is less than the diffraction coherence length. In this case, diffraction will probe lattice parameters that are averaged over multiple microdomains. Groups of microdomains will then be perceived as a macrodomain of a structurally homogeneous (microdomain-averaged) phase. To resolve the tetragonal phase, high resolution diffraction conditions are required [18].

Consider a stress-accommodating macrodomain plate that consists of two different variants of tetragonal microdomains formed by the stress-free transformation strains [9,10], $\boldsymbol{\varepsilon}(1)$ and $\boldsymbol{\varepsilon}(3)$, respectively. These microdomains are $(101)_{c}$ twin related and form alternating layers along the $(101)_{c}$ plane. If the tetragonal strains are small, the microdomain-averaged stress-free strain is

$$
\begin{aligned}
\langle\boldsymbol{\varepsilon}(\omega)\rangle & =\omega \boldsymbol{\varepsilon}(1)+(1-\omega) \boldsymbol{\varepsilon}(3) \\
& =\omega\left(\begin{array}{ccc}
\varepsilon_{3} & 0 & 0 \\
0 & \varepsilon_{1} & 0 \\
0 & 0 & \varepsilon_{1}
\end{array}\right)+(1-\omega)\left(\begin{array}{ccc}
\varepsilon_{1} & 0 & 0 \\
0 & \varepsilon_{1} & 0 \\
0 & 0 & \varepsilon_{3}
\end{array}\right),
\end{aligned}
$$

where $\varepsilon_{3}=\left(c_{t}-a_{c}\right) / a_{c}$ and $\varepsilon_{1}=\left(a_{t}-a_{c}\right) / a_{c}, c_{t}$ and $a_{t}$ are the crystal lattice constants of the tetragonal phase, $a_{c}$ is the lattice constant of the cubic phase, and $\omega$ is the volume fraction of the microdomains with transformation strain $\boldsymbol{\varepsilon}(1)$ in the macrodomain plate.

The microdomain-averaged crystal lattice has pseudoorthorhombic symmetry. In fact, the crystal lattice is monoclinic, however, the monoclinic distortion is negligible since $\left|\varepsilon_{1}\right| \ll 1$ and $\left|\varepsilon_{3}\right| \ll 1$. The lattice parameters of this mixed state are

$$
\begin{aligned}
& a_{\mathrm{ad}}=a_{t}+\left(c_{t}-a_{t}\right) \omega, \quad b_{\mathrm{ad}}=a_{t}, \\
& c_{\mathrm{ad}}=c_{t}-\left(c_{t}-a_{t}\right) \omega,
\end{aligned}
$$

which, by definition, are directed along the $\langle 100\rangle_{c}$ axes. As follows From Eq. (3), the parameters $\left(a_{\mathrm{ad}}, b_{\mathrm{ad}}, c_{\mathrm{ad}}\right)$ are self-adjusted parameters that strongly depend on $\omega$. The value $\omega$ may significantly vary because application of electric field or applied stress will result in microdomain rearrangement that increases the volume fraction of favorably oriented microdomains. However, as follows from Eq. (3), the sum of the self-adjustable parameters $a_{\mathrm{ad}}+c_{\mathrm{ad}}$ and the value $b_{\mathrm{ad}}$,

$$
a_{\mathrm{ad}}+c_{\mathrm{ad}}=a_{t}+c_{t}, \quad b_{\mathrm{ad}}=a_{t},
$$

are not independent parameters: they are expressed in terms of the intrinsic parameters $a_{t}$ and $c_{t}$ of the tetragonal phase forming microdomains. Therefore, Eq. (4) provides invariance conditions: their left-hand sides are invariants, irrespective of any changes in $a_{\mathrm{ad}}$ and $c_{\mathrm{ad}}$. Below we call the conditions (4) the general invariance conditions.

General invariance follows from stress accommodation, requiring elimination of misfits along the $(101)_{c}$ boundaries between microdomains within a macrodomain. However, this is insufficient to fully eliminate the transformation-induced stress. Complete stress accommodation occurs when the microdomain-averaged crystal lattice parameters of the product phase are related to the parameters of the parent phase by the IPS [12,13,15-17]. To do this, the microdomain structure must adjust further to eliminate also the stress generated by misfits along the habit plane of the macrodomains. To achieve this condition, the system must choose a special value of the geometrical parameter, $\omega=\omega_{0}=\varepsilon_{1} /\left(\varepsilon_{1}-\varepsilon_{3}\right)=$ $\left(a_{t}-a_{c}\right) /\left(a_{t}-c_{t}\right)$, that provides an IPS. Substituting $\omega_{0}$ into Eq. (3) provides the lattice parameters of the completely stress-accommodated microdomain-averaged state:

$$
a_{\mathrm{ad}}=a_{c}, \quad b_{\mathrm{ad}}=a_{t}, \quad c_{\mathrm{ad}}=c_{t}+a_{t}-a_{c} .
$$

It is important to realize that the relations are a particular case of the relations (3). Below we will call the conditions (5) the special invariance conditions. The special constraint (5) is lifted under applied fields, affecting the value of the geometrical parameter $\omega$. However, under 
external fields, the general invariance conditions (4) are still preserved.

The observation of a phase whose crystal lattice parameters are dependent on those of the parent cubic phase and those forming microdomains (i.e., no independence or uniqueness) is the fingerprint of an adaptive state $[9,10]$. This fingerprint has been shown to be followed [9] in martensitic states [19,20].

Both the general (4) and special (5) invariance conditions should be preserved with changing temperature and composition. Temperature dependent neutron diffraction data have previously been obtained for various PMN- $x$ PT specimens [3], in the compositional range of $31 \%<x<37 \%$. These investigations were performed under zero electric field. Thus, any change in the invariance conditions can be attributed solely to the effect of temperature. The comparison of the data presented in Fig. 2 confirms our prediction that the monoclinic phase is an adaptive phase composed of tetragonal phase microdomains. Over significant temperature and composition ranges, the lattice parameters of this monoclinic phase $\left(a_{m}, b_{m}, c_{m}\right)$ are shown to obey both the general and special invariance conditions of the adaptive phase as

$$
a_{m}+c_{m}=a_{t}+c_{t}, \quad b_{m}=a_{t}
$$
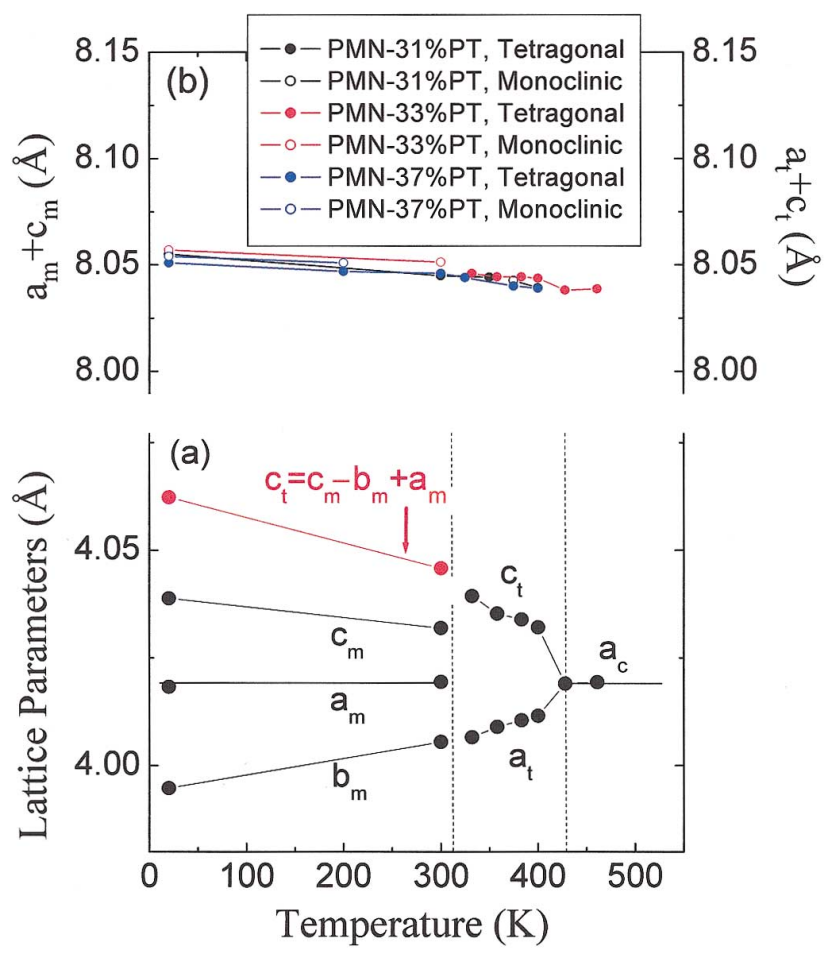

FIG. 2 (color). (a) Temperature dependent lattice parameters for PMN-33\%PT; these data were taken by neutron diffraction by Noheda et al. [3]. The points and lines in red are the lattice parameters calculated by Eq. (7). (b) Temperature dependence of the general invariance condition of $a_{m}+c_{m}=a_{t}+c_{t}$. Data are shown for PMN-31\%PT, PMN-33\%PT, and PMN-37\%PT.

$$
a_{m}=a_{c}, \quad b_{m}=a_{t}, \quad c_{m}=c_{t}+a_{t}-a_{c} .
$$

Figure 2(a) shows the temperature dependence of the lattice constants for PMN-33\% PT (similar results were found for PMN-31\%PT and PMN-37\%PT [3], but are not shown). The tetragonal lattice parameters $\left(a_{t}, c_{t}\right)$ and cubic lattice parameter $\left(a_{c}\right)$ are shown in the figure. At lower temperatures, a $\mathrm{FE}_{m}$ phase with $c_{m} \neq b_{m} \neq a_{m}$ is present. The special "invariance" conditions (7) were found to be well maintained. To illustrate this, the lattice parameter $c_{t}$ of the metastable $\mathrm{FE}_{t}$ phase within the stability field of the $\mathrm{FE}_{m}$ phase was calculated using $c_{t}=$ $c_{m}-b_{m}+a_{m}$, which can be obtained through Eq. (7). The calculated values of $c_{t}$ are plotted in red, alongside the measured ones of the $\mathrm{FE}_{m}$ phase. It can be seen in Fig. 2(a) that the calculated parameter $c_{t}=c_{m}-b_{m}+$ $a_{m}$ provides a continuous extension of the lattice parameter $c_{t}$ measured in the stability field of the $\mathrm{FE}_{t}$ phase. Also, in accordance with the special invariance conditions (7), the lattice parameter $b_{m}$ of the $\mathrm{FE}_{m}$ phase is a continuous extension of the lattice parameter $a_{t}$ of the $\mathrm{FE}_{t}$ phase. Furthermore, the lattice parameter $a_{m}$ is equal to $a_{c}$; however, this parameter does not continuously extend between the cubic and $\mathrm{FE}_{m}$ phases. Rather, it undergoes hibernation in the $\mathrm{FE}_{t}$ phase, spontaneously reappearing in the $\mathrm{FE}_{m}$ phase.

Figure 2(b) shows the general invariance conditions as a function of temperature for various PMN- $x \mathrm{PT}$ compositions, $a_{m}+c_{m}=a_{t}+c_{t}$. The data clearly demonstrate that the general invariance conditions (6) are obeyed over the wide temperature $(20<T<500 \mathrm{~K})$ and composition $(31 \%<x<37 \%)$ ranges of the $\mathrm{FE}_{m}$ phase. To be noted is the fact that the plot shows a small negative slope. This is due to the observed temperature dependence of the lattice parameters of the tetragonal phase.

Application of electric field violates the special invariance conditions (7). However, the theory predicts that general invariance conditions (6) will still be fulfilled. The field dependence of the crystal lattice parameters of PZN-8\%PT have been determined by single crystal neutron diffraction [4], as shown in Fig. 3(a). Figure 3(b) shows the corresponding plot of a combination of $a_{m}+$ $c_{m}$ following from general invariance conditions (6). The results clearly demonstrate that the general invariance conditions are rigidly obeyed over the entire $\mathrm{FE}_{m}$ stability range. This demonstrates that the changes in the lattice parameters with electric field are due to a redistribution of microdomains. The $\mathrm{FE}_{t}$ phase is reached when the crystal has been fully detwinned by field, which occurs when the geometric ratio in Eq. (3) is $\omega=0$. The adaptive phase theory predicts a gradual rotation of the microdomain-averaged polarization under electric field. This is caused by electric-field-induced changes in $\omega$. The polarization of the adaptive phase is $\mathbf{P}_{\mathrm{ad}}=\omega\left(P_{t}, 0,0\right)+$ $(1-\omega)\left(0,0, P_{t}\right)=P_{t}(\omega, 0,1-\omega)$, which rotates in the (010) $)_{c}$ plane. 


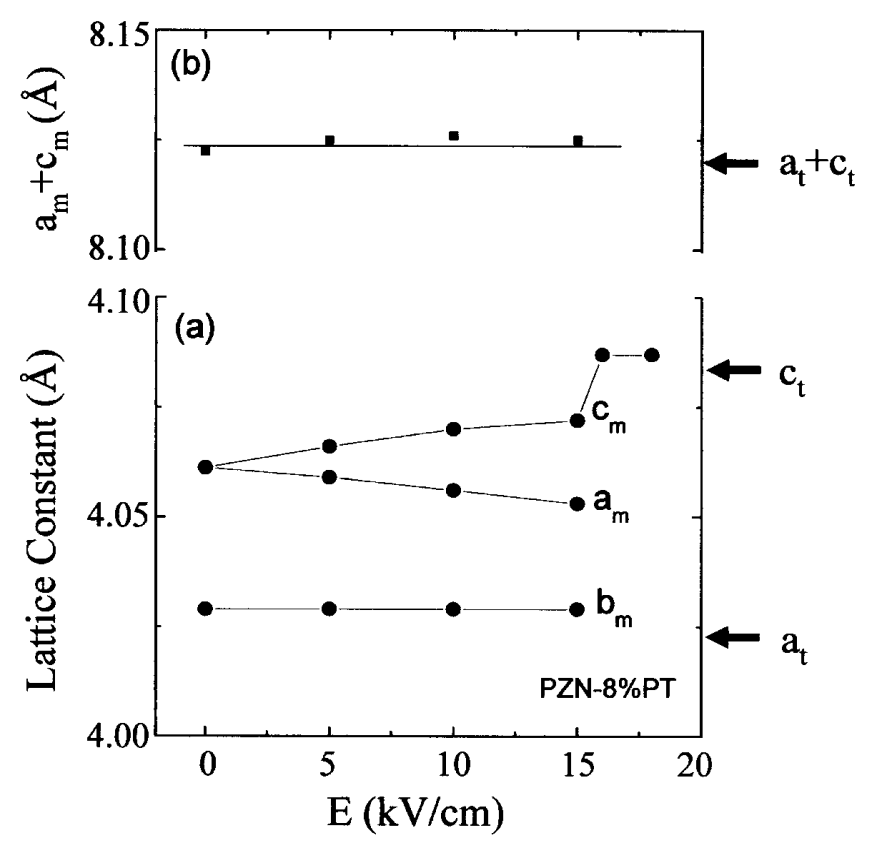

FIG. 3. (a) Dependence of the crystal lattice parameters $c_{m}$, $a_{m}$, and $b_{m}$ of the pseudo-orthorhombic (monoclinic) phase in PZN-8\%PT on the applied electric field [4]. (b) Dependence of the sum $a_{m}+c_{m}$ on electric field $\mathbf{E}$. The independence of this sum on $\mathbf{E}$ is the fulfillment of the invariance condition of Eq. (6). The results demonstrate that $a_{m}+c_{m}=a_{t}+c_{t}$ as required by the general invariance condition.

The results clearly show that the general invariance conditions (6) and the more restrictive special invariance conditions (7) are obeyed over a broad range of temperatures and compositions (Fig. 2). The general invariance conditions (6) are also fulfilled under applied field (Fig. 3). This continued fulfillment of the invariance conditions unambiguously demonstrates that the $\mathrm{FE}_{m}$ phase is not a homogeneous (unique) phase requiring independent lattice parameters. Rather the $\mathrm{FE}_{m}$ phase can be described over its entire stability range as an adaptive mixed state with microdomain-averaged adjustable topological parameters and polarization, given by the invariance conditions. These invariance conditions imposed on the crystal lattice parameters are so restrictive and special that it is nearly impossible that their continued fulfillment over an entire temperature, concentration, electric field, and stress stability ranges of an intermediate phase is coincidental.
Finally, it is important to note that stress-accommodating rhombohedral microdomains also result in an adaptive monoclinic phase. We find that the monoclinic phases formed by tetragonal and rhombohedral microdomains coincide with the $M_{C}$ type in [3] and the $M_{A}$ type in [5], respectively. The theory of adaptive monoclinic phases composed of rhombohedral microdomains will be published separately.

Y. M. J., Y. U.W., and A. G. K. gratefully acknowledge the NSF support under Grant No. DMR-0242619. J. F. L. and D.V. gratefully acknowledge the ONR support under Grants No. N000140210340, No. N000140210126, and No. MURI N000140110761.

[1] B. Noheda et al., Phys. Rev. Lett. 86, 3891 (2001).

[2] K. Ohwada et al., J. Phys. Soc. Jpn. 70, 2778 (2001).

[3] B. Noheda et al., Phys. Rev. B 66, 054104 (2002).

[4] B. Noheda et al., Phys. Rev. B 65, 224101 (2002).

[5] D. Vanderbilt and M. Cohen, Phys. Rev. B 63, 094108 (2001).

[6] H. Fu and R. Cohen, Nature (London) 403, 281 (2000).

[7] A. Garcia and D. Vanderbilt, Appl. Phys. Lett. 72, 2981 (1998).

[8] L. Bellaiche, A. Garcia, and D. Vanderbilt, Phys. Rev. Lett. 84, 5427 (2000).

[9] A. G. Khachaturyan, S. M. Shapiro, and S. Semenovskaya, Phys. Rev. B 43, 10832 (1991).

[10] D. Viehland, J. Appl. Phys. 88, 4794 (2000).

[11] Z. Xu et al., Philos. Mag. A 74, 395 (1996).

[12] M. S. Wechsler, D. S. Lieberman, and T. A. Read, J. Met. 197, 1503 (1953).

[13] J. C. Bowles and J. K. Mackenzie, Acta Metall. 2, 129 (1954).

[14] V. I. Syutkina and E. S. Jakovleva, Phys. Status Solidi 21, 465 (1967).

[15] A. G. Khachaturyan and G. A. Shatalov, Zh. Eksp. Teor. Fiz. 56, 1037 (1969) [Sov. Phys. JETP 29, 557 (1969)].

[16] A. G. Khachaturyan, The Theory of Structural Transformations in Solids (Wiley, New York, 1983).

[17] A. L. Roytburd, Fiz. Tverd. Tela 10, 3619 (1968) [Sov. Phys. Solid State 10, 2870 (1969)].

[18] A. G. Khachaturyan, Kristallografia (USSR) 4, 646 (1959) (in Russian).

[19] S. M. Shapiro et al., Phys. Rev. Lett. 62, 1298 (1989).

[20] H. Seto, Y. Noda, and Y. Yamada, J. Phys. Soc. Jpn. 59, 965 (1990). 